\title{
Percutaneous Renal Biopsy Using an 18-Gauge Automated Needle Is Not Optimal
}

\author{
George Sousanieh William L. Whittier Roger A. Rodby Vasil Peev \\ Stephen M. Korbet \\ Division of Nephrology, Department of Internal Medicine, Rush University Medical Center, Chicago, IL, USA
}

\section{Keywords}

Renal biopsy · Kidney biopsy · Needle gauge · Adequacy · Complications

\begin{abstract}
Background: As percutaneous renal biopsies (PRBs) are increasingly performed by radiologists, an increase in the use of 18-gauge automated needle stands to compromise adequacy. We compare the adequacy and safety of PRB with 14-, 16-, and 18-gauge automated needles. Methods: PRB of native (N-592) and transplant $(\mathrm{T}-1,023)$ kidneys was performed from January 2002 to December 2019 using real-time ultrasound. Baseline clinical and laboratory data, biopsy data (number of cores, total glomeruli, and total glomeruli per core), and outcome (hematoma on renal US at 1-h, complications, and transfusion) were collected prospectively. PRB with N14g (337) versus N16g (255) and T16g (892) versus T18g (131) needles were compared. A $p$ value of $<0.05$ was significant. Results: PRB with an 18-g needle yielded the lowest number of total glomeruli per biopsy (N14g vs. N16g: 33 \pm 13 vs. $29 \pm 12, p<0.01$ and T16g vs. T18g: $34 \pm 16$ vs. $21 \pm$ $11, p<0.0001)$, significantly fewer total glomeruli per core (T16g vs. T18g: $12.7 \pm 6.4$ vs. $9.6 \pm 5.0, p<0.001$ and N16g vs. T18g: $14.2 \pm 6.3$ vs. $9.6 \pm 5.0, p<0.001)$. A hematoma by renal US 1 -h post-PRB was similar for native $(14 \mathrm{~g}-35 \%$ vs. $16 \mathrm{~g}-$
\end{abstract}

$29 \%, p=0.2$ ), and transplant biopsies (16g-10\% vs. $18 \mathrm{~g}-9 \%$, $p=0.9)$ and the complication rate for native $(14 \mathrm{~g}-8.9 \%$ vs. $16 \mathrm{~g}-7.1 \%, p=0.5)$, transplant biopsies (16g-4.6\% vs. $18 \mathrm{~g}-$ $1.5 \%, p=0.2)$ and transfusion rate for native $(14 \mathrm{~g}-7.7 \%$ vs. $16 \mathrm{~g}-5.8 \%, p=0.4$ ), and transplant biopsies (16g-3.8\% vs. $18 \mathrm{~g}-0.8 \%, p=0.1$ ) were similar irrespective of needle size. Conclusions: PRB of native and transplant kidneys with the use of a 16-gauge needle provides an optimal sample. However, our experience in transplant biopsies suggests the use of an 18-gauge needle stands to jeopardize the diagnostic accuracy of the PRB while not improving safety.

(c) 2021 S. Karger AG, Basel

\section{Introduction}

The percutaneous renal biopsy (PRB) has remained an invaluable tool for nephrologists for over 60 years [1]. The success of the PRB has resulted from the ability to safely obtain an adequate sample of tissue/glomeruli allowing a diagnosis and prognosis which then guides therapeutic decisions. Obtaining an "adequate" sample is of particular importance in lesions that are "focal" in nature. Thus, it has been shown that obtaining at least 20 glomeruli in a biopsy specimen is optimal, minimizing the risk of a misdiagnosis [2-6].

karger@karger.com
www.karger.com/ajn
Karger $V^{\prime /}$


With the introduction of the automated renal biopsy needle in 1990, the technical ease of performing renal biopsies was greatly improved without an increased risk of complication compared to manual needles [1, 7]. While a 14-gauge needle was initially used, due to concerns over higher complication rates, there was an increasing trend to using the smaller 16-gauge needle [1, 8, 9], which has been shown to provide an equally adequate sample [913].

Over the past 10 years, as the trend for performing PRB has transitioned from nephrologist to interventional radiologist $[9,14,15]$, we have seen the increasing use of the smaller, 18-gauge needle [9]. The use of this smaller needle stands to jeopardize the diagnostic value of the biopsy if an adequate number of glomeruli is not obtained. To enhance our understanding of the impact of the 18-gauge needle on the success of PRB, we evaluated our single academic center's experience with the use of automated 14-, 16-, and 18-gauge needles in PRB performed in adults with real-time ultrasound.

\section{Methods}

A PRB was performed in 1,615 adult ( $\geq 15$ years) patients (native renal biopsy-592 and transplant renal biopsy-1,023) from January 2002 to December 2019 at Rush University Medical Center. An attending nephrologist or a fellow under the direct supervision of an attending nephrologist performed all biopsies. Imaging was performed by an experienced radiologist using real-time ultrasound as previously described [1,16,17]. A 14-gauge (native-337), 16-gauge (native-255 and transplant-892), or 18-gauge (transplant-131) automated biopsy needle (CR Bard Inc., Covington, GA, USA) was used at the discretion of the attending nephrologist. This study was approved by the institutional review board at Rush University Medical Center.

Information collected at the time of biopsy included age, gender, race, systolic and diastolic blood pressures (BP), serum $\mathrm{Cr}$ (SCr), activated partial thromboplastin time (PTT: normal $\leq 33.0$ s), and hemoglobin ( $\mathrm{Hgb}$ ) concentration. The needle gauge and number of biopsy cores obtained (both determined by the nephrologist performing the procedure) were recorded at the time of the biopsy.

The biopsy specimens were collected by a pathology lab technician and viewed under a dissecting microscope. The specimens are then divided for light microscopy (LM), immunofluorescence microscopy (IF), and electron microscopy (EM) evaluation in native renal biopsies and for LM and IF in transplant biopsies. The number of glomeruli obtained by LM, IF, and EM was recorded for each biopsy. The number of glomeruli observed on the tissue for LM, IF, and EM was determined by the nephropathologist and recorded in their report. The total number of glomeruli per biopsy was determined by adding the number of glomeruli on LM + IF + EM for native biopsies and LM + IF for transplant biopsies. The number of glomeruli per core was calculated by dividing the total glomeruli by the number of cores obtained.

Percutaneous Renal Biopsy Adequacy and Needle Gauge
Immediately following the biopsy, patients were closely monitored and at 1-h post-PRB, the biopsied kidney was examined by ultrasound for the presence of a perinephric hematoma. Patients undergoing native renal biopsies were then admitted to the hospital for $23 \mathrm{~h}$ of observation, while patients undergoing transplant biopsies were monitored for 8-h post biopsy and then discharged home if stable as previously described $[17,18]$.

Patients were prospectively evaluated for the development and timing of a post-PRB complication. A clinically significant complication was defined by the presence of gross hematuria, a perinephric hematoma, and/or a drop in Hgb resulting in the need for an intervention (i.e., transfusion of blood products or radiographic or surgical procedure) or the need for readmission or death.

\section{Statistical Analysis}

Given the differences in the technical performance and postbiopsy observation, statistical analysis based on needle gauge was initially performed for native and transplant biopsies. To determine the differences in the total number of glomeruli obtained, the number of cores obtained and the number of glomeruli per core using 14-, 16-, and 18-gauge biopsies, both native and transplant biopsy results were then analyzed. Statistical analysis was performed using the Mann-Whitney and Kruskal-Wallis tests for continuous data or the Fisher's exact and $\chi^{2}$ tests for categorical data. Data are reported as mean \pm standard deviation, and a $p$ value of $<0.05$ was considered significant.

\section{Results}

\section{Baseline Features}

The baseline demographic, clinical, and laboratory data based on type of biopsy (native or transplant renal biopsy) and needle gauge are shown in Table 1 . Fellows performed $90 \%$ of the native and $57 \%$ of the transplant biopsies. The majority of native biopsies (57\%) were done using a 14-gauge needle, and the majority of transplant biopsies (87\%) utilized a 16-gauge needle. Patients undergoing a native renal biopsy with a 14 -gauge versus a 16-gauge needle were similar in age and gender and had similar systolic and diastolic BPs at the time of the biopsy. The SCr, PTT, and Hgb levels pre-biopsy were similar as well.

Patients undergoing a transplant renal biopsy with a 16 -gauge versus 18 -gauge needle were more often female but were similar in age. They also had similar systolic and diastolic BPs, as well as SCr, PTT, and Hgb values prebiopsy.

\section{Biopsy Adequacy}

The biopsy adequacy data based on type of biopsy (native or transplant renal biopsy) and needle gauge are shown in Table 2 . In native renal biopsies, the average number of cores obtained was higher in patients biop- 
Table 1. Baseline demographic, clinical, and laboratory features

\begin{tabular}{|c|c|c|c|c|c|c|}
\hline & \multicolumn{3}{|l|}{ Native } & \multicolumn{3}{|l|}{ Transplant } \\
\hline & 14-gauge & 16-gauge & $p$ value & 16-gauge & 18-gauge & $p$ value \\
\hline$N(\%)$ & $337(57)$ & $255(43)$ & & $892(87)$ & $131(13)$ & \\
\hline Fellow biopsy, $n(\%)$ & $316(94)$ & $215(84)$ & 0.0002 & $551(62)$ & $31(24)$ & $<0.0001$ \\
\hline Age, years & $47 \pm 17$ & $46 \pm 18$ & 0.41 & $51 \pm 14$ & $53 \pm 15$ & 0.15 \\
\hline Female, $n(\%)$ & $148(44)$ & $106(42)$ & 0.62 & $440(49)$ & $42(32)$ & 0.0002 \\
\hline \multicolumn{7}{|l|}{$\mathrm{BP}$} \\
\hline Systolic, mm Hg & $133 \pm 19$ & $134 \pm 19$ & 0.63 & $141 \pm 22$ & $142 \pm 18$ & 0.32 \\
\hline Diastolic, mm Hg & $78 \pm 12$ & $80 \pm 13$ & 0.16 & $78 \pm 13$ & $80 \pm 13$ & 0.02 \\
\hline $\mathrm{SCr}, \mathrm{mg} / \mathrm{dL}$ & $2.3 \pm 2.2$ & $2.2 \pm 2.3$ & 0.06 & $3.0 \pm 1.7$ & $2.6 \pm 1.4$ & 0.01 \\
\hline PTT normal & $305 / 336(91 \%)$ & $234 / 255(92 \%)$ & 0.76 & $59 / 684(9 \%)$ & $4 / 113(4 \%)$ & 0.087 \\
\hline Pre-Hgb, g/dL & $11.7 \pm 2.1$ & $11.4 \pm 2.2$ & 0.19 & $11.1 \pm 1.7$ & $11.7 \pm 2.1$ & 0.01 \\
\hline
\end{tabular}

BP, blood pressure, PTT, partial thromboplastin time; Hgb, hemoglobin.

Table 2. Biopsy adequacy

\begin{tabular}{|c|c|c|c|c|c|c|}
\hline & \multicolumn{3}{|l|}{ Native } & \multicolumn{3}{|l|}{ Transplant } \\
\hline & 14-gauge & 16-gauge & $p$ value & 16-gauge & 18-gauge & $p$ value \\
\hline$N$ & 337 & 255 & & 892 & 131 & \\
\hline Cores & $2.3 \pm 0.7$ & $2.2 \pm 0.6$ & 0.03 & $2.8 \pm 0.7$ & $2.2 \pm 0.6$ & $<0.0001^{*}$ \\
\hline \multicolumn{7}{|l|}{ Total glomeruli } \\
\hline$(\mathrm{LM}+\mathrm{IF}+\mathrm{EM})$ & $33 \pm 13$ & $29 \pm 12$ & 0.003 & $34 \pm 16$ & $21 \pm 11$ & $<0.0001^{* *}$ \\
\hline$\geq 20$ & $286(85 \%)$ & $209(82 \%)$ & 0.37 & $740(83 \%)$ & $60(46 \%)$ & $<0.0001^{* * *}$ \\
\hline Total gloms/core & $15.4 \pm 7.7$ & $14.2 \pm 6.3$ & 0.099 & $12.7 \pm 6.4$ & $9.6 \pm 5.0$ & $<0.0001^{* * * *}$ \\
\hline
\end{tabular}

LM, light microscopy; IF, immunofluorescence microscopy; EM, electron microscopy. * N14 versus N16, $p>$ 0.05 ; N14 versus T16, $p<0.001$; N14 versus T18, $p>0.05$; N16 versus T16, $p<0.001$; N16 versus T18, $p>0.05$; T16 versus T18, $p<0.001$. ** N14 versus N16, $p<0.05$; N14 versus T16, $p>0.05 ;$ N14 versus T18, $p<0.001 ;$ N16 versus T16, $p<0.01 ; \mathrm{N} 16$ versus T18, $p<0.001$; T16 versus T18, $p<0.001$. ${ }^{* * *} \mathrm{~N} 14$ versus N16, P 0.37 ; N14 versus T16, P 0.44; N14 versus T18, $p<0.0001$; N16 versus T16, P 0.07; N16 versus T18, $p<0.0001$; T16 versus T18, $p<0.0001{ }^{* * * *}$ N14 versus N16, $p>0.05$; N14 versus T16, $p<0.001$; N14 versus T18, $p<0.001$; N16 versus T16, $p<0.001 ; \mathrm{N} 16$ versus T18, $p<0.001 ; \mathrm{T} 16$ versus T18, $p<0.001$.

sied with a 14-gauge versus a 16-gauge needle, and the total number of glomeruli per biopsy on average was higher as well. The number of biopsies with $\geq 20$ glomeruli and the total glomeruli per core were similar. In transplant renal biopsies, the average number of cores obtained was higher in patients biopsied with a 16-gauge versus an 18-gauge needle, and the total number of glomeruli per biopsy on average was higher as well. The number of biopsies with $\geq 20$ glomeruli and the total glomeruli per core were significantly less in biopsies done with the 18-gauge needle.
The proportion of biopsies with $\geq 20$ total glomeruli was similar among the transplant biopsies performed with a 16 -gauge needle ( $83 \%$ ) compared to native biopsies performed with a 14 -gauge $(85 \%)$ or 16 -gauge $(82 \%)$ needle but was significantly less among those performed with an 18-gauge needle (46\%) compared to biopsies with 14- or 16-gauge needle. The total number of glomeruli per core was also significantly less with the 18-gauge needle (9.6 \pm 5.0$)$ compared to transplant biopsies with a 16-gauge needle (12.7 \pm 6.4$)$ and native biopsies with 14-gauge (15.4 \pm 7.7$)$ or 16-gauge (14.2 \pm 6.3) needle. 
Table 3. Post-biopsy hematoma by US at $1 \mathrm{~h}$

\begin{tabular}{lccl}
\hline Native biopsy & 14-gauge & 16-gauge & $p$ value \\
\hline Yes, $n(\%)$ & $84(35)$ & $74(29)$ & 0.18 \\
No & 156 & 181 & \\
\hline Total & 240 & 255 & \\
\hline Transplant biopsy & 16-gauge & 18 -gauge & $p$ value \\
\hline Yes, $n(\%)$ & $61(10)$ & $12(9)$ & 0.87 \\
No & 634 & 119 & \\
\hline Total & 695 & 131 & \\
\hline
\end{tabular}

Post-Biopsy Hematoma at 1-h and Complications

A post-biopsy hematoma by renal ultrasound at 1-h (Table 3) was similar in native biopsies (14-gauge, 35\% vs. 16 -gauge, 29\%) and transplant biopsies (16-gauge, 10\% vs. 18-gauge, $9 \%$ ) irrespective of needle size. The postbiopsy complications and transfusion rate are shown in Table 4. In native biopsies, the complication rate (14-gauge, $8.9 \%$ vs. 16-gauge, $7.1 \%$ ) and transfusion rate (14-gauge, $7.7 \%$ vs. 16 -gauge, $5.8 \%$ ) were not significantly different irrespective of needle size. Similarly, in transplant biopsies, the complication rate (16-gauge, $4.6 \%$ vs. 18 -gauge, $1.5 \%$ ) and transfusion rate (16-gauge, $3.8 \%$ vs. 18 -gauge, $0.8 \%$ ) were not significantly different irrespective of needle size.

\section{Discussion}

We find that percutaneous transplant renal biopsy in adults using real-time ultrasound and automated needles has a glomerular yield that risks being suboptimal when performed with an 18-gauge needle compared with a 16-gauge needle and was not associated with a significantly lower post biopsy rate of hematomas, complications, or transfusions. Additionally, we confirm our previous observation [13] that PRB of native kidneys using a 16 -gauge needle has a glomerular yield that is similar to biopsies performed with a 14-gauge needle.

The success of the PRB has resulted from the ability to safely obtain an adequate number of glomeruli allowing a diagnosis which will then guide management decisions. The importance of sample size cannot be underestimated when evaluating glomerular lesions which are focal in nature and whose prognosis depends on the degree of involvement (i.e., focal segmental glomerulosclerosis, lupus

Percutaneous Renal Biopsy Adequacy and Needle Gauge
Table 4. Biopsy complication requiring intervention

\begin{tabular}{lccc}
\hline Native biopsy & 14-gauge & 16-gauge & $p$ value \\
\hline$N$ & 337 & 255 & \\
Complications, $n(\%)$ & $30(8.9)$ & $18(7.1)$ & 0.45 \\
Transfusions, $n(\%)$ & $26(7.7)$ & $15(5.8)$ & 0.42 \\
\hline Transplant biopsy & 16-gauge & 18 -gauge & $p$ value \\
\hline$N$ & 892 & 131 & \\
Complications, $n(\%)$ & $41(4.6)$ & $2(1.5)$ & 0.16 \\
Transfusions, $n(\%)$ & $34(3.8)$ & $1(0.8)$ & 0.07 \\
\hline
\end{tabular}

nephritis, vasculitis, and crescentic glomerulonephritis) $[2,5]$. A biopsy containing only 10 glomeruli has a $35 \%$ probability of missing the lesion if the prevalence of diseased glomeruli in the kidney is $10 \%$, but the probability decreases to $12 \%$ if 20 glomeruli are contained in the biopsy. Thus, it has been recommended that obtaining at least 20 glomeruli in a biopsy specimen is optimal, minimizing the risk of a misdiagnosis [2-6].

Numerous studies of PRB of native and transplant kidneys $[1,9,19-25]$ have shown that when an 18-gauge needle is used the sample size is significantly smaller and the quality of the sample (the number of intact glomeruli) is poorer. In PRB of native kidneys, Kim et al. [20] found that the average number of glomeruli per core was 9.9 with an 18-gauge needle compared to 15.3 with a 14-gauge needle. In PRB of transplant kidneys, Nicholson et al. [21] also demonstrated that using an 18-gauge needle resulted not only in a significantly smaller sample size compared to biopsies done with a 16- or 14-gauge needle ( 9 vs. 11 vs. 15 glomeruli) but was also associated with less diagnostic success ( 53 vs. 76 vs. $85 \%$ ). The study by Tondel et al. [9] found that the sample size was significantly smaller in native kidney biopsies performed with 18-gauge needles, a median of 9 glomeruli per biopsy, compared to 12 glomeruli with either the 16- or 14-gauge needles. Mai et al. [25] also demonstrated fewer glomeruli in native kidney biopsies performed with 18-gauge automated needles compared to 16-gauge needles (median: 12 vs. 19 glomeruli, $p<0.001)$ despite having significantly more cores per biopsy (median: 3 vs. 2 cores, $p<0.001$ ). And recently, in a multicenter study, Peters et al. [26] reported fewer glomeruli per biopsy core in biopsies performed with 18 -gauge needles compared to 16 -gauge needles in both native ( 8 vs. 11 glomeruli per core, $p<0.001$ ) and transplant biopsies ( 8 vs. 12 glomeruli per core, $p$ 0.02). While 
PRB with an 18-gauge needle was only performed on transplant biopsies in our study, the number of glomeruli per core we observed was consistent with findings in previous studies in PRB of native and transplant kidneys. Additionally, the glomerular yield we observed with the use of 16-gauge needles in PRB of both native and transplant kidneys was also consistent with prior studies and similarly, in transplant biopsies, demonstrated a higher yield than with 18 -gauge needles.

Since the introduction of the automated needles, there has been an increasing tendency to use smaller, 18-gauge, needles to perform PRB $[1,8,9,22]$. The reason for this may be due to the increasing number of biopsies being performed by radiologists as they tend to prefer using 18-gauge needles $[9,14,15,22]$. There has been a steady transition in the performance of PRB from nephrologist to interventional radiologist over the past 30 years. In our own program, while $100 \%$ of nephrologists who trained from 1984 to 1990 performed PRB after their fellowship, this steadily declined to only $20 \%$ of nephrologists completing training after 2011 [14]. Berns [27] found that only $20 \%$ of 30 nephrologists trained in the University of Pennsylvania Health System fellowship program in the last 6 years were performing PRB. Similarly, Yuan et al. [28], in a survey of 55 practicing nephrologists trained at Walter Reed National Military Medical Center from 1985 to 2017 found that only 35\% performed PRB. Since 2012, as a result of the complex and time-consuming process of coordinating and performing the PRB, the Walter Reed nephrology program refers all PRBs to interventional radiology [28].

Gupta and Balogun [22] found that an 18-gauge needle was used in $69 \%$ of biopsies performed by a radiologists compared to only $13 \%$ of biopsies performed by Nephrologists. Tondel et al. [9] found that overall radiologists performed the biopsy in $54 \%$ of cases with nephrologists performing only $33 \%$ of biopsies and that $62 \%$ of biopsies were done using an 18-gauge needle. Over a 22-year period, there was an increase in both the number of biopsies done by radiologists as well as the proportion of biopsies done using 18-gauge needles. In the study by Mai et al. [25], all of the biopsies performed with a 16-gauge needle were done by nephrologist, and all performed with an 18 -gauge needle were done by radiologist.

The rationale for using the smaller 18-gauge needle is unclear but may be due to the perception that it is safer with fewer complications compared to larger gauge needles [29]. However, in the prospective trial by Nicholson et al. [21] in transplant biopsies, no differences in complication rates were found for biopsies done with 14-, 16-, or 18-gauge automated needles. Similarly, numerous studies have found no differences in complication rates or transfusion requirements based on needle size $[9,10,12,25,30$, 31]. Roth et al. [31] evaluated 149 native PRBs done over a 1-year period using 16- and 18-gauge automated needles and found the post-PRB transfusion rate to be no difference between the needles (4.9 and 6.0\%, respectively). Similarly, while a meta-analysis of PRB of native kidneys by Corapi et al. [8] demonstrated the rate of transfusion was significantly greater in patients biopsied with a 14-gauge automated needle (2.1\%), it was no different for biopsies using either a 16- or 18-gauge needle ( 0.6 vs. $0.5 \%)$. Mai et al. [25] also found no significant difference in perinephric hematomas ( 2.0 vs. $2.2 \%, p=0.77$ ), transfusion rates ( 1.1 vs. $0 \%, p=0.37$ ), or overall complication rates (3.7 vs. $2.2 \%, p=0.49)$ for biopsies done with a 16 - or 18-gauge needle in native kidneys. Peters et al. [26] reported no significant difference in major complications in biopsies performed with 16-gauge needles compared to 18 -gauge needles in both native (6.7 vs. $6.8 \%)$ and transplant biopsies (4.8 vs. 3.4\%). Thus, based on previous findings in native and transplant biopsies, the use of an 18 -gauge needle was not associated with a decreased risk of complication. In our study, the experience with an 18-gauge needle was limited to PRB of transplants and was not associated with a lower risk of complication than with a 16-gauge needle. As a result, our study cannot address the safety of an 18-gauge needle in native kidney biopsies.

One final observation from our study is the decreased rate of complication associated with PRB of transplant kidneys relative to native biopsies. This is consistent with our previous experience comparing $\mathrm{PRB}$ of transplant and native kidneys [18] and has been reported by others as well $[26,32]$. A number of reasons have been used to explain the lower complication rate, but most notable is the markedly different anatomic approach to a transplant biopsy compared to a native kidney biopsy. The superficial position of the transplanted kidney allows easier access to the kidney as well as the ability to directly apply pressure and tamponade the biopsy site post-PRB.

In conclusion, $\mathrm{PRB}$ of native and transplant kidneys with the use of a 16-gauge needle provides an optimal sample. However, our experience in transplant biopsies suggests the use of an 18-gauge needle stands to jeopardize the diagnostic accuracy of the PRB while not improving safety.

\section{Conflict of Interest Statement}

The results presented in this paper have not been published previously in whole or part, except in abstract form. 


\section{Funding Sources}

The authors did not receive any funding.

\section{Author Contributions}

George Sousanieh, MD: conception, design, and analysis and interpretation of data. Drafting the article and revising it. Providing intellectual content of critical importance to the work described. Final approval of the version has to be published. William L. Whittier, MD: conception, design, and analysis and interpreta- tion of data. Revising the manuscript and providing intellectual content of critical importance to the work described. Final approval of the version has to be published. Roger A. Rodby, MD: analysis and interpretation of data. Revising the article and providing intellectual content of critical importance to the work described. Final approval of the version has to be published. Vasil Peev, MD: revising the article and providing intellectual content of critical importance to the work described. Final approval of the version has to be published. Stephen M. Korbet, MD: conception, design, and analysis and interpretation of data. Drafting the article and revising it. Providing intellectual content of critical importance to the work described. Final approval of the version has to be published.

\section{References}

1 Korbet SM. Percutaneous renal biopsy. Semin Nephrol. 2002;22(3):254-67.

2 Wang HJ, Kjellstrand CM, Cockfield SM, Solez K. On the influence of sample size on the prognostic accuracy and reproducibility of renal transplant biopsy. Nephrol Dial Transplant. 1998;13(1):165-72.

3 Oberholzer M, Torhorst J, Perret E, Mihatsch MJ. Minimum sample size of kidney biopsies for semiquantitative and quantitative evaluation. Nephron. 1983;34(3):192-5.

4 Madaio MP. Renal biopsy. Kidney Int. 1990; 38(3):529-43.

5 Corwin HL, Schwartz MM, Lewis EJ. The importance of sample size in the interpretation of the renal biopsy. Am J Nephrol. 1988;8(2): 85-9.

6 Luciano RL, Moeckel GW. Update on the native kidney biopsy: core curriculum 2019. Am J Kidney Dis. 2019;73(3):404-15.

7 Wiseman DA, Hawkins R, Numerow LM, Taub KJ. Percutaneous renal biopsy utilizing real time, ultrasonic guidance and a semiautomated biopsy device. Kidney Int. 1990; 38(2):347-9.

8 Corapi KM, Chen JL, Balk EM, Gordon CE. Bleeding complications of native kidney biopsy: a systematic review and meta-analysis. Am J Kidney Dis. 2012;60(1):62-73.

9 Tondel C, Vikse BE, Bostad L, Svarstad E. Safety and complications of percutaneous kidney biopsies in 715 children and 8,573 adults in Norway 1988-2010. Clin J Am Soc Nephrol. 2012;7:1591-7.

10 Bataille S, Jourde N, Daniel L, Mondain JR, Faure M, Gobert P, et al. Comparative safety and efficiency of five percutaneous kidney biopsy approaches of native kidneys: a multicenter study. Am J Nephrol. 2012;35(5):387-93.

11 Simard-Meilleur MC, Troyanov S, Roy L, Dalaire E, Brachemi S. Risk factors and timing of native kidney biopsy complications. Nephron Extra. 2014;4(1):42-9.

12 Manno C, Strippoli GF, Arnesano L, Bonifati C, Campobasso N, Gesualdo L, et al. Predictors of bleeding complications in percutaneous ultrasound-guided renal biopsy. Kidney Int. 2004;66(4):1570-7.
13 Chunduri S, Whittier WL, Korbet SM. Adequacy and complication rates with 14- vs. 16-gauge automated needles in percutaneous renal biopsy of native kidneys. Semin Dial. 2015;28(2):E11-4.

14 Korbet SM, Whittier WL, Rodby RA. Changing trends in the performance of percutaneous renal biopsy from nephrologist to interventional radiologist: a single-center experience. Am J Nephrol. 2018;48(5):326-9.

15 Whittier WL, Korbet SM. Who should perform the percutaneous renal biopsy: a nephrologist or radiologist? Semin Dial. 2014; 27(3):243-5.

16 Birnholz JC, Kasinath BS, Corwin HL. An improved technique for ultrasound guided percutaneous renal biopsy. Kidney Int. 1985; 27(1):80-2.

17 Korbet SM, Volpini KC, Whittier WL. Percutaneous renal biopsy of native kidneys: a single-center experience of 1,055 biopsies. Am J Nephrol. 2014;39(2):153-62.

18 Whittier WL, Gashti C, Saltzberg S, Korbet S. Comparison of native and transplant kidney biopsies: diagnostic yield and complications. Clin Kidney J. 2018;11(5):616-22.

19 Doyle AJ, Gregory MC, Terreros DA. Percutaneous native renal biopsy: comparison of a $1.2 \mathrm{~mm}$ spring- loaded system with a traditional $2 \mathrm{~mm}$ hand-driven system. Am J Kidney Dis. 1994;23:498-503.

20 Kim D, Kim H, Shin G, Ku S, Ma K, Shin S, et al. A randomized, prospective, comparative study of manual and automated renal biopsies. Am J Kidney Dis. 1998;32(3):426-31.

21 Nicholson ML, Wheatley TJ, Doughman TM, White SA, Morgan JD, Veitch PS, et al. A prospective randomized trial of three different sizes of core-cutting needle for renal transplant biopsy. Kidney Int. 2000;58(1):390-5.

22 Gupta RK, Balogun RA. Native renal biopsies: complications and glomerular yield between radiologists and nephrologists. J Nephrol. 2005; 18:553-8.
23 Walker PD. The renal biopsy. Arch Pathol Lab Med. 2009;133(2):181-8.

24 Arora K, Punia RS, D'Cruz S. Comparison of diagnostic quality of kidney biopsy obtained using $16 \mathrm{G}$ and $18 \mathrm{G}$ needles in patients with diffuse renal disease. Saudi J Kidney Dis Transpl. 2012;23(1):88-92.

25 Mai J, Yong J, Dixson H, Makris A, Aravindan A, Suranyi MG, et al. Is bigger better? a retrospective analysis of native renal biopsies with 16 gauge versus 18 gauge automatic needles. Nephrology. 2013;18(7):525-30.

26 Peters B, Mölne J, Hadimeri H, Hadimeri U, Stegmayr B. Sixteen gauge biopsy needles are better and safer than 18 gauge in native and transplant kidney biopsies. Acta Radiol. 2017; 58(2):240-8.

27 Berns JS. Training nephrology fellows in temporary hemodialysis catheter placement and kidney biopsies is needed and should be required. Clin J Am Soc Nephrol. 2018;13(7): 1099-101.

28 Yuan CM, Nee R, Little DJ, Narayan R, Childs JM, Prince LK, et al. Survey of kidney biopsy clinical practice and training in the United States. Clin J Am Soc Nephrol. 2018;13(5): 718-25.

29 Gupta S, Wallace MJ, Cardella JF, Kundu S, Miller DL, Rose SC. Quality improvement guidelines for percutaneous needle biopsy. J Vasc Interv Radiol. 2010;21(7):969-75.

30 McMahon GM, McGovern ME, Bijol V, Benson CB, Foley R, Munkley K, et al. Development of an outpatient native kidney biopsy service in low-risk patients: a multidisciplinary approach. Am J Nephrol. 2012;35(4): 321-6.

31 Roth R, Parikh S, Makey D, Foster J, Rozenblit G, Satoskar A, et al. When size matters: diagnostic value of kidney biopsy according to the gauge of the biopsy needle. Am J Nephrol. 2013;37(3):249-54

32 Aaltonen S, Finne P, Honkanen E. Outpatient kidney biopsy: a single center experience and review of literature. Nephron. 2020;144(1): 14-20. 